\title{
MapReduce rationality verification based on object Petri net
}

\author{
DING Zeliu1,2,", GUO Deke ${ }^{3}$, CHEN Xi ${ }^{4}$, and CHEN Jin ${ }^{1}$ \\ 1. School of Electronics Engineering, Navy University of Engineering, Wuhan 430033, China; \\ 2. Research Center of Evaluation and Demonstration, Academy of Military Sciences, Beijing 100091, China; \\ 3. College of Systems Engineering, National University of Defense Technology, Changsha 410073, China; \\ 4. School of Computer Science, McGill University, Montreal H3A 2A7, Canada
}

\begin{abstract}
As a powerful distributed data processing mechanism, MapReduce supports abundant parallel applications that process massive data on computer clusters. To process the massive data efficiently and correctly, a rational design for the MapReduce procedure is desired. An irrational MapReduce procedure can cause great waste of computing resources and even paralyze the execution system. With the wide application of MapReduce, the unavoidable drawback of irrational MapReduce procedures becomes increasingly serious. To solve this problem, a method for verifying the rationality of a MapReduce procedure before executing it on a computer cluster is proposed. This method constructs the rationality criteria for MapReduce, and then studies an automatic approach for modelling MapReduce with an executable model object Petri net (OPN). Finally, the approaches for automatically identifying the rationality criteria by analyzing the consequence of model execution is developed. The results from extensive case studies demonstrate that the proposed method is feasible and effective.
\end{abstract}

Keywords: MapReduce, rationality verification, cloud computing framework.

DOI: $10.21629 /$ JSEE.2019.05.05

\section{Introduction}

MapReduce, proposed by Google [1], has become one of the most typical and widely used distributed computing and cloud computing mechanisms [2,3]. A MapReduce procedure can process terabytes of data rapidly by separating a complex computation into Map tasks and Reduce tasks, which are performed in parallel on hundreds or even thousands of computing nodes in a large-scale computer cluster. The availability of open-source MapReduce programs, such as OpenStack [4] and Hadoop [5], make more and more internet service corporations provide various MapReduce services.

\footnotetext{
Manuscript received September 15, 2017.

*Corresponding author.

This work was supported by the Natural Science Foundation of Hubei Province, China (2016CFB287).
}

Although Google's MapReduce mechanism provides a framework for classifying and merging huge amounts of raw data, it cannot ensure the rationality of a specific MapReduce procedure, which refers to the ability that the MapReduce procedure can be executed according to the designed work flow and accomplished in expected time.

The expansion of MapReduce applications drives the emergence of various irrational problems caused by designing errors in the original MapReduce procedures. If a MapReduce procedure is found to be irrational during the executing process on thousands of computing nodes, it would cause a tremendous waste of computing resources.

One example is the "straggler" problem [6]. A "straggler" is a Map or Reduce task whose execution time is extremely longer than that of other tasks. In a MapReduce procedure, Reduce tasks cannot be carried out until all Map tasks are finished, and the whole procedure ends only when all the Reduce tasks have been achieved. Accordingly, hundreds of computing nodes might have to wait for a single straggler, which will cause two serious negative impacts. First, the MapReduce itself will not be accomplished successfully at the cost of long period of waiting. Second, the occupied computing nodes are unavailable for other service requests, hence new tasks at those computing nodes may accumulate rapidly.

An irrational MapReduce procedure can lead to congestion in the computer cluster, and interferes other services running on the cluster. Even worse, the whole cluster may be paralyzed. With the rapid and wide application of MapReduce, the unavoidable drawback of irrational MapReduce procedures becomes increasingly serious.

To address this problem, the rationality of a complex MapReduce procedure should be verified before executing it on a computer cluster. Although many efforts have been done on congestion control and resource allocation for computer clusters $[7,8]$, they are all based upon the consideration of network operation and maintenance. The ex- 
isting work, however, pays less attention to verifying the rationality of a MapReduce procedure before execution. One reason is that the majority of the irrational problems in a MapReduce procedure are difficult to be directly quantified or formally described. The more important reason is that a MapReduce procedure, which is designed as running on a cluster, cannot be verified by running on a single computer, and the cluster may have already suffered resource waste once an irrational problem is found.

Our research objective is to study a method to automatically estimate whether the design of a MapReduce procedure is rational before executing it on a computer cluster, so that all its operations at Map and Reduce phases can be efficiently and correctly performed. By this method, the designers can earlier detect the irrational problems to avoid resource waste and system paralysis. Thus, the network efficiency and service benefits can be improved.

Our method is based on a representative executable graphical model named object Petri net (OPN) [9,10]. It is used for simulating and analyzing the whole running process of dynamical systems. Due to the difficulties to directly identify those irrational problems in a MapReduce procedure, we model MapReduce operations with OPN and find those irrational problems by analyzing the running situations of the resulting model. A distinguished property of OPN is its hierarchical structure. In the hierarchical structure, any object in an OPN model represents another OPN model. Consequently, OPN can well model the inner hierarchical structure of a complex MapReduce procedure and simulate its running process. Our research group has built a modeling and simulation environment for OPN, which can not only execute OPN in a visual way, but also evaluate and analyze the execution results.

To the best of our knowledge, this is the first work studying a method for rationality verification of MapReduce. The main innovations of this work are as follows:

(i) We explore the main rationality criteria as the correct principles for verifying the rationality of a MapReduce procedure.

(ii) We study an approach for modelling MapReduce with OPN. As MapReduce and OPN are expressed in different ways, we present the formal descriptions of MapReduce and OPN. We then present an approach to automatically model a MapReduce procedure with OPN using the formal descriptions as input. Further, we provide an algorithm to validate the correctness of the resulting model.

(ii) We develop the approaches for automatically identifying the rationality criteria of MapReduce by analyzing the consequence of model execution. The results of case studies prove that our approaches are feasible and effective.
The rest of this paper is organized as follows. Section 2 introduces the background and related work. Section 3 explores the rationality criteria of MapReduce. Section 4 studies the formal descriptions of MapReduce and OPN. Section 5 proposes the approach to modelling MapReduce with OPN. Section 6 shows how to identify the rationality criteria of MapReduce by analyzing the consequence of model execution. Section 7 illustrates the effectiveness of our method. Section 8 concludes this paper.

\section{Background and related work}

\subsection{Background}

With a simple and practical processing procedure, MapReduce provides a standard mechanism for distributed data processing. A complex MapReduce procedure processes terabytes of data through a sequence of jobs, and each job consists of one Map phase and one Reduce phase. Each phase includes multiple parallel Map or Reduce tasks. Map tasks are responsible for data classification and preparation of intermediate data for Reduce tasks. Reduce tasks are responsible for merging the intermediate data and generating output values, which are sent to the distributed file systems or the Map tasks of the next job.

In a computer cluster, MapReduce let a master server control many worker servers to perform Map and Reduce tasks [1]. Fig. 1 illustrates a MapReduce procedure with only one job.

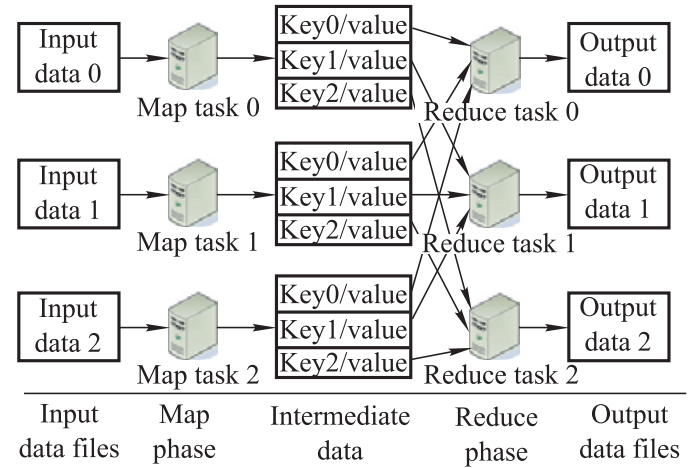

Fig. 1 Procedure of a MapReduce job

\subsection{Related work}

As the expansion of MapReduce applications, the basic operations of MapReduce no longer meet diverse user requirements and expose a few problems. The existing work focused on improving MapReduce and finding ways to verify those problems $[11,12]$.

The straggler problem was firstly proposed by Google in [1], where the stragglers were only attributed to hardware faults, such as bad disks, memory failures, and so on [13]. It was suggested to re-perform straggler tasks on the other 
computing nodes. This scheme, however, cannot avoid the stragglers caused by irrational MapReduce procedures.

In order to reduce the response time of MapReduce executed by Hadoop, Zaharia et al. [14] proposed an improved stochastic method by estimating the execution time of Map and Reduce tasks. Kavulya et al. [15] utilized the Gini coefficient to analyze large amounts of statistical data about the tasks and compared the results with threshold values to identify stragglers. These two methods can find stragglers only after executing MapReduce procedures and cannot predict stragglers in advance.

In [6], it was illustrated that the straggler problem of MapReduce could be caused by designing errors, i.e., unreasonably distributing input or intermediate data. It also emphasized the importance of verifying the straggler problem for designing an efficient MapReduce. However, it did not present any method for verifying stragglers.

Ren et al. presented Hooper [16], a speculation-aware job scheduler, for mitigating the impact of stragglers. By coordinating speculation and scheduling decisions, Hooper reduces the average job completion time by up to $50 \%-$ $66 \%$. However, Hooper ignores the dependencies between jobs, and can be implemented only when the tasks of jobs have been running on clusters. Moreover, Hooper can hardly troubleshoot the stragglers caused by irrationally designed procedures.

Although the straggler problem has received particular attention, the rationality verification of MapReduce still lacks a feasible method to address all of those problems.

Wei et al. [17] proposed a framework for MapReduce to ensure the service integrity of data processing. It presented an integrity verification method for executing MapReduce. This method, however, assumed that the MapReduce procedures were rationally designed and could be executed correctly on computer clusters.

In order to debug faults in datacenter networks, Zhu et al. [18] presented a network telemetry system, which works by tracing specific packets and sending their copies to different analysis servers to detect the potential faults. This packet-level network telemetry has been demonstrated to be effective, but cannot predict the irrational MapReduce procedures causing network faults before running on datacenter networks.

The procedures of MapReduce have been modified recently for some particular applications. These modifications add some pretreatment or assistant operations to the Map and Reduce phases. Yang et al. [19] added a Merge operation after the Reduce phase to further merge the result data. Kruijf et al. [20] added three pretreatment steps before the Reduce phase to facilitate the Reduce operations for generating more accurate results.

\section{Rationality criteria}

We explore three main rationality criteria for MapReduce as follows. These criteria are the foundations for identifying the major problems in an irrationally designed MapReduce procedure.

\subsection{No straggler}

In order to guarantee that the Map and Reduce tasks can be executed smoothly and the whole procedure is not interrupted, we set no straggler as the first rationality criterion.

As mentioned above, a straggler is a Map or Reduce task which takes relatively much longer execution time than the others. The existence of a straggler has negative impacts on the computer cluster. Since the straggler problem caused by hardware faults has been addressed in [1], this paper only focuses on the straggler problem caused by designing errors, which is more difficult to detect. From a MapReduce design perspective, a straggler is created by an unreasonable distribution of input or intermediate data [6].

A Map straggler appears if the input data need to be divided into many types of intermediate data and each type of intermediate data has a large number of key/value pairs. Taking word count $[1,21]$ as an example, if a Map task needs to divide the Complete Works of Shakespeare into each word as a key of intermediate data, and all the other Map tasks only deal with short novels, then the former one will become a potential Map straggler. A Reduce straggler appears if it has to merge plenty of intermediate data coming from many Map tasks. For example, if a Reduce task needs to merge the numbers of each word in the Complete Works of Shakespeare, and all the other Reduce tasks only deal with the intermediate data divided from short novels, then the former one will become a potential Reduce straggler. For more information about word count, we suggest readers to refer to Section 5.4 or literatures [1] and [21].

\subsection{No Map conflict}

In order to ensure that all intermediate data can be transmitted correctly, we set no Map conflict as the second rationality criterion.

In a MapReduce job, if one or more Map tasks send intermediate data with the same key to different Reduce tasks, we call this phenomenon the Map conflict. Taking word count for example again, if all Map tasks send the numbers of some words to a Reduce task except one Map task sending the number of the words to another Reduce task, then the words cannot be counted correctly.

A Map conflict is mainly resulted from the inconsistent operations. The design of a complex MapReduce usually needs the collaboration of many designers. Different de- 
signers may assign the same type of intermediate data to different Reduce tasks by mistake. A Map conflict prevents the intermediate key/value pairs with the same key from being merged completely. Although a Map conflict may not interrupt the running of MapReduce, it causes an inaccurate result.

\subsection{Reasonable execution time}

In order to evaluate whether the execution time of MapReduce can meet user's requirement, we set reasonable execution time as the third rationality criterion.

The users as well as the service providers may care more about this criterion, since they usually pursue the financial profits by enhancing the execution efficiency.

The total execution time of a MapReduce procedure is composed by the in/out (I/O) time for reading and writing data and the running time of the Map and Reduce tasks. Usually, the larger amount of input data brings longer I/O time. Moreover, when the size of input data is fixed, the total execution time is determined by the number of Map and Reduce tasks. Increasing the number of tasks can decrease the load of each computer node and the execution time, because more nodes handle the MapReduce procedure.

\section{Formal description}

We provide the formal descriptions of MapReduce and OPN for automatically modelling a MapReduce procedure with OPN. In this way, we can verify the rationality of a MapReduce procedure through quantitative methods.

\subsection{Formal description of MapReduce}

The components of a basic MapReduce procedure include Map tasks, Reduce tasks, and assignments. Map and Reduce tasks are two types of parallel data processing operations running on computing nodes by means of predesigned programs. An assignment is the transmission of intermediate data from a Map or Reduce task to another task.

Definition 1 The formal description of MapReduce

$$
\text { MapReduce }=(M A ; R E ; A S)
$$

Let $M A=\left\{\operatorname{Map}_{m} \mid 0 \leqslant m<M\right\}$ denote the set of Map tasks.

Let $R E=\left\{\right.$ Reduce $\left._{r} \mid 0 \leqslant r<R\right\}$ denote the set of Reduce tasks.

Let $A S=\left\{\operatorname{Assign}_{a} \mid 0 \leqslant a<A\right\}$ denote the set of assignments.

For ease of explanation, let Task $x$ denote a Map or Reduce task, having the same attributes as those of $\mathrm{Map}_{m}$ or Reduce $_{r}$.

\subsection{Formal description of OPN}

As an advanced Petri net $[22,23]$, the major improvement of OPN is the hierarchical structure where each object (called sub-object in the rest of this paper) in an OPN can be another OPN. With the hierarchical structure, OPN can easily model a complex process consisting of various situations.

Besides sub-objects, the components of an OPN model include places, transitions, tokens, and directed arcs $[9,10]$. A place represents the state of a stage during the whole process modeled by OPN. There are three types of places, the normal place, the input place and the output place. A transition represents the operation that causes a stage change. A token represents the information or data transmitted between two different operations. The tokens drive the execution of an OPN model. OPN works by the movements of tokens from one place to another through the transition or sub-object between them. A directed arc is used for interconnecting a place and a transition or sub-object. In an OPN model, directed arcs show the ways for the token movements. Fig. 2 illustrates an example of the OPN model.

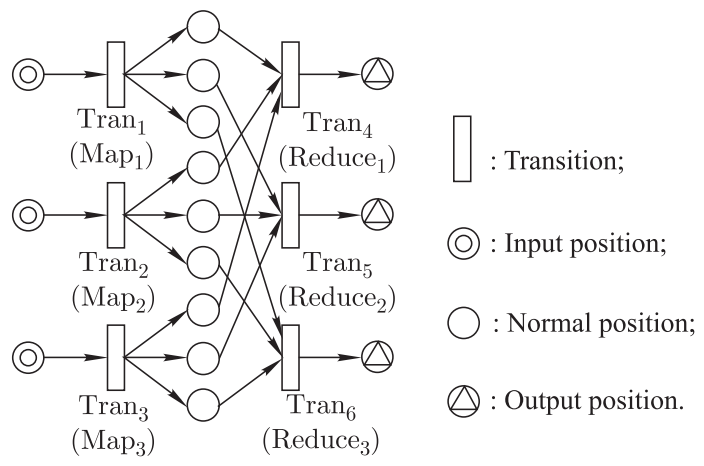

Fig. 2 OPN model of Case 1

Definition 2 The formal description of OPN

$$
\mathrm{OPN}=(P O ; T R ; S u b O)
$$

Let $P O=\left\{\right.$ Place $\left._{p} \mid 0 \leqslant p<P\right\}$ denote the set of places.

Let $T R=\left\{\operatorname{Tran}_{t} \mid 0 \leqslant t<T\right\}$ denote the set of transitions.

Let $S u b O=\left\{\right.$ SubObject $\left._{s} \mid 0 \leqslant s<S\right\}$ denote the set of sub-objects.

For ease of explanation, let $\mathrm{TrOb}_{i}$ denote a transition or sub-object, having the same attributes as those of $\operatorname{Tran}_{t}$


tioned description of OPN can be regarded as the formal description of a sub-object in a higher level OPN.

\section{Modelling MapReduce with OPN}

In this section, we provide an approach for automatically modelling a MapReduce procedure with OPN. 
Besides OPN, several technologies can be used for modelling complex procedures, such as standard Petri net, Opnet and finite state automaton (FSA). Table 1 compares their suitability for simulating the execution of MapReduce. Standard Petri net and FSA are typical simulation tools for system processes, but they can hardly model the hierarchy of MapReduce operations. Opnet is mainly applied to simulate network environments, including network topologies, equipments and protocols, and has no correspondence with MapReduce.

Table 1 Comparison of suitability for simulating the execution of MapReduce

\begin{tabular}{ccccc}
\hline Suitability & OPN & $\begin{array}{c}\text { Standard } \\
\text { Petri Net }\end{array}$ & OPNET & FSA \\
\hline Elements correspondence & Yes & Yes & No & No \\
Hierarchical structure & Yes & No & Yes & No \\
Process modelling & Yes & Yes & No & Yes \\
Visual modelling & Yes & Yes & Yes & Yes \\
\hline
\end{tabular}

We leverage OPN to model a MapReduce procedure not only for its inheritance of the property of standard Petri net, but also for the following two reasons.

The first one is that the elements of MapReduce are in one-to-one correspondence with that of OPN, namely the elements of MapReduce play the similar roles as that of OPN. The tasks of MapReduce and the transitions of OPN both represent the operations of the processes modelled by MapReduce and OPN, respectively. The keys of MapReduce and the tokens of OPN both represent the data transmitted between two operations, which trigger the corresponding operations to start. Although the places of OPN represent different states, they can be regarded as the transmissions of information since they assign tokens to certain transitions. Hence, the assignments of MapReduce correspond to the places of OPN.

The second one is that our lab has developed an OPN modelling and simulation environment (OPNMSE), which is specialized in describing and executing OPN models. After transforming a MapReduce procedure into an OPN model by program languages according to the following principles, OPNMSE can read the XML format of the obtained OPN model and generate its graphic format. Moreover, OPNMSE can set the token arrival rate for each place according to a certain probability distribution. That simulates the execution time for each task. Then we can easily analyze the running of the obtained OPN model with OPNMSE, so as to determine the rationality of the MapReduce procedure.

\subsection{Approach to modelling MapReduce with OPN}

The basic principles of modelling MapReduce with OPN are presented as follows: (i) The tasks of MapReduce are transformed into the transitions or sub-objects of OPN.

Let $C$ denote the threshold on the number of keys processed by a task, to determine whether it is a potential straggler. According to the verification criteria, if the types of intermediate keys processed by the task are larger than $C$, it may be a potential straggler and needs to be transformed into a sub-object. Otherwise, this task needs to be transformed into a transition.

(ii) The assignments of MapReduce are transformed into the normal places of OPN.

For any assignment Assign $_{a}$, if the tasks sending and receiving Assign ${ }_{a}$ have been transformed into $\mathrm{TrOb}_{i}$ and $\mathrm{TrOb}_{j}$, respectively, Assign ${ }_{a}$ is transformed into the normal place between $\mathrm{TrOb} i$ and $\mathrm{TrOb}_{j}$.

(iii) The keys of MapReduce are transformed into the tokens of OPN.

If an assignment has been transformed into a place, then the token capacity of the place is set to be the number of types of intermediate keys sent by the assignment.

(iv) The connection relationships between tasks and assignments of MapReduce are transformed into the directed arcs of OPN.

If an assignment Assign $_{a}$ has been transformed into a normal place Place petween $^{\mathrm{TrO} O b_{i}}$ and $\mathrm{TrOb}_{j}$, the relationship between Assign ${ }_{a}$ and the task sending Assign ${ }_{a}$ is transformed into a directed arc from $\mathrm{TrOb} b_{i}$ to $\mathrm{Place}_{p}$, the relationship between Assign $n_{a}$ and the task receiving Assign $_{a}$ is transformed into a directed arc from Place $_{p}$ to $\mathrm{TrOb}_{j}$.

(v) Adding input and output places to the OPN.

The whole procedure of a MapReduce task can be transformed into a preliminary OPN model according to above principles. The resulting OPN, however, is inexecutable due to the lack of input and output places. Input places work as the start of OPN. Only when each input place has a token, OPN can start to run. Output places work as the end of OPN. Only when each output place has received a token, the execution of OPN can be finished.

The places performed before and after $\mathrm{TrOb}_{i}$ are called the anterior and posterior places of $\mathrm{TrOb} b_{i}$, respectively. For any transition or sub-object $\mathrm{TrOb}_{i}$, if there is no anterior place, we add an input place and a directed arc from the input place to $\mathrm{TrOb}_{i}$. If there is no posterior place, we add an output place and a directed arc from $\mathrm{TrOb}_{i}$ to the output place.

\subsection{Approach to building a sub-object}

In order to determine whether a Map or Reduce task represented by a sub-object is a potential straggler, we should further build an OPN model for the sub-object. Let 


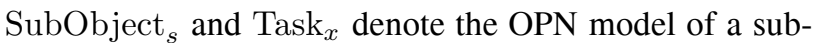
object and the Map or Reduce task it represents, respectively. The OPN model of a sub-object is achieved through the following steps.

(i) The first step is adding input and output places to the sub-object.

The number of input (or output) places in SubObject $_{s}$ is equal to the amount of input (or output) assignments of Task $_{x}$. The token capacity of every input or output place in SubObject ${ }_{s}$ is equal to the amount of keys of the corresponding assignment. If Task ${ }_{x}$ has no input (or output) assignment, we need to add one input (or output) place with token capacity as 1 to SubObject $_{s}$.

(ii) The second step is adding input and output transitions to the sub-object.

Here input (or output) transitions refer to the transitions connecting with the input (or output) places of SubObject $_{s}$. Each input (or output) transition connects with an input (or output) place. In our work, input and output transitions do not represent any operation of MapReduce. They are used for completing the structure of the sub-object.

(iii) The third step is adding operational transitions to the sub-object.

We use several transitions, called operational transitions, to represent the concrete operations of Task ${ }_{x}$. Let $\mid$ Task $_{x}$.Keys $\mid$ denote the number of types of intermediate keys processed by Task ${ }_{x}$. Let $Y$ denote the number of operational transitions, which is determined by $\mid$ Task $_{x}$.Keys $\mid$ and the threshold number $C$, then $Y$ is given by

$$
Y=\left\lceil\frac{\mid \operatorname{Task}_{x} \cdot \text { Keys } \mid}{C}\right\rceil \text {. }
$$

Since $\mid \operatorname{Task}_{x}$.Keys $\mid$ is larger than $C$, the ceiling of $C$ dividing $\mid$ Task $_{x}$. Keys $\mid$ is an integer larger than 2 .

(iv) The final step is adding normal places and directed arcs to the sub-object.

Normal places are used for transmitting tokens from input transitions to operational transitions and from operational transitions to output transitions. There is a normal place connecting each pair of input and operational transitions through two arcs, and so does each pair of operational and output transitions. For each input (or output) transition, the total token capacity of its normal places is equal to that of its input (or output) place. Moreover, if the number of its normal places is larger than the token capacity of its input (or output) place, the token capacity of its every normal place is 1 . This ensures that each normal place in the subobject can be performed. The difference between the token capacities of any two normal places connecting to the same input or output transition is no more than 1 .

\subsection{Validation}

Let Place $_{p}$.Type denote the type of Place $_{p}$, and Place $_{p}$. Type $\in$ \{Normal, Input, Output $\}$. Let $\mathrm{TrOb}_{i}$. forePlace and $\mathrm{TrOb}_{i}$.postPlace denote the anterior and posterior places of $\mathrm{TrOb}{ }_{i}$, respectively. Let Task $_{x}$.foreAssign and Task ${ }_{x}$.postAssign denote the anterior and posterior assignments of Task ${ }_{x}$, respectively. To ensure that the resulting OPN correctly represents the original MapReduce, we validate the modelling from three aspects indicated by the pseudocode of Algorithm 1. Since the number of transitions is much larger than that of subobjects, the time complexity of Algorithm 1 is $O\left(T^{2}\right)$.



Lines 1-2: The total number of transitions and subobjects in OPN should be equal to that of Map and Reduce tasks in MapReduce.

Lines 3-10: The number of input places should be equal to that of the Map tasks without input assignments. In addition, the number of output places should be equal to that of the Reduce tasks without output assignments.

Lines $11-17$ : There is only one place between each pair of transitions or sub-objects.

\subsection{Case studies}

\subsubsection{Case 1: word count}

Word count $[1,21]$ is a simple case of MapReduce application. It includes one Map phase and one Reduce phase. The Map tasks send the words and their occurrences as the keys and values of intermediate data, respectively. The Reduce tasks calculate the total number of occurrences of every word as its count. 
We employ a word count case to calculate the amount of personal pronouns, interrogative pronouns and singular reflexive pronouns in a document. It includes three Map tasks and three Reduce tasks. Every Map task reads a part of the document, and sends each kind of personal pronouns, interrogative pronouns, singular reflexive pronouns and their corresponding occurrences to the three Reduce tasks, respectively. The Reduce tasks separately sum together all counts for each kind of personal pronouns, interrogative pronouns and singular reflexive pronouns. Since there are 12 types of personal pronouns, five types of interrogative pronouns and five types of singular reflexive pronouns, the numbers of keys processed by the Reduce tasks are 12, 5 and 5 , respectively.

\subsubsection{Case 2: enumerating triangles}

In order to prove that our method is reasonable for complex MapReduce with multi-jobs, we employ the famous problem of enumerating triangles [24] as the second case. Enumerating triangles is a MapReduce application in graph theory used for enumerating all the triangles in a large graph. It includes four Map phases and four Reduce phases.

According to [24], we use a graph with 10 vertices and 12 edges, as shown in Fig. 3, to illustrate the MapReduce procedure. The input file records the edges of the graph, and every edge is represented by its two vertices.

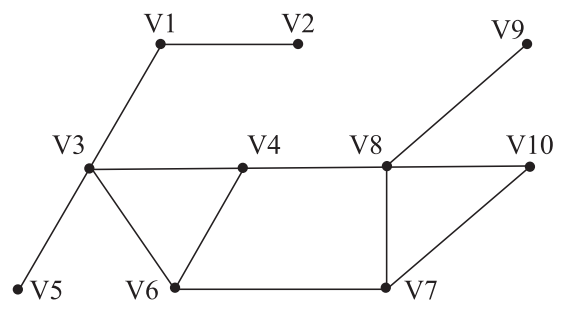

Fig. 3 Example graph for Case 2

The first Map phase includes three tasks, which send the vertices and their adjacent edges as intermediate keys and values. The first Reduce phase includes two tasks, each of which processes five types of keys to calculate the degree of each vertex.

The second Map phase includes two tasks, which send the edges and the degree of a vertex for each edge as intermediate keys and values. The second Reduce phase includes three tasks, each of which processes four types of keys to calculate the degrees of the two vertices of each edge.

The third Map phase includes three tasks, which send the low-degree vertex of each edge and the edges as intermediate keys and values, respectively. The third Reduce phase includes two tasks, each of which processes five types of keys to calculate every pair of adjacent edges whose common vertex is their low-degree vertex.

The fourth Map phase includes two tasks, which send the edges as intermediate keys and values. They also send the two nonadjacent vertices of the obtained adjacent edges and these adjacent edges as intermediate keys and values. Finally, the fourth Reduce phase includes three tasks, each of which processes four types of keys to calculate the triangles.

\subsubsection{Modelling MapReduce procedures}

Table 2 and Table 3 present the abbreviated formal descriptions of Case 1 and Case 2, respectively. In these tables, From and To denote the tasks that send and receive the assignments, respectively.

Table 2 Assignments of Case 1

\begin{tabular}{|c|c|c|c|c|}
\hline$A S$ & From & To & Keys & $\mid$ Keys \\
\hline Assign $_{1}$ & $\mathrm{Map}_{1}$ & Reduce $_{1}$ & Personal pronouns & 12 \\
\hline $\operatorname{Assign}_{2}$ & $\mathrm{Map}_{1}$ & Reduce $_{2}$ & Interrogative pronouns & 5 \\
\hline $\operatorname{Assign}_{3}$ & $\mathrm{Map}_{1}$ & Reduce $3_{3}$ & Singular reflexive pronouns & 5 \\
\hline $\operatorname{Assign}_{4}$ & $\mathrm{Map}_{2}$ & Reduce $_{1}$ & Personal pronouns & 12 \\
\hline $\operatorname{Assign}_{5}$ & $\mathrm{Map}_{2}$ & Reduce $_{2}$ & Interrogative pronouns & 5 \\
\hline Assign $_{6}$ & $\mathrm{Map}_{2}$ & Reduce $_{3}$ & Singular reflexive pronouns & 5 \\
\hline Assign $_{7}$ & $\mathrm{Map}_{3}$ & Reduce $_{1}$ & Personal pronouns & 12 \\
\hline $\operatorname{Assign}_{8}$ & $\mathrm{Map}_{3}$ & Reduce $_{2}$ & Interrogative pronouns & 5 \\
\hline Assign $_{9}$ & $\mathrm{Map}_{2}$ & Reduce $_{3}$ & Singular reflexive pronouns & 5 \\
\hline \multicolumn{5}{|c|}{ Table 3 Assignments of Case 2} \\
\hline$A S$ & From & To & Keys & $\mid \overline{\text { Keys }}$ \\
\hline $\operatorname{Assign}_{1}$ & $\mathrm{Map}_{1}$ & Reduce $_{1}$ & $v 1, v 2, v 3, v 4, v 5$ & 5 \\
\hline $\operatorname{Assign}_{2}$ & $\mathrm{Map}_{1}$ & Reduce $_{2}$ & $v 6, v 7, v 8, v 9, v 10$ & 5 \\
\hline Assign $_{3}$ & $\mathrm{Map}_{2}$ & Reduce $_{1}$ & $v 1, v 2, v 3, v 4, v 5$ & 5 \\
\hline $\operatorname{Assign}_{4}$ & $\mathrm{Map}_{2}$ & Reduce $_{2}$ & $v 6, v 7, v 8, v 9, v 10$ & 5 \\
\hline $\operatorname{Assign}_{5}$ & $\mathrm{Map}_{3}$ & Reduce $_{1}$ & $v 1, v 2, v 3, v 4, v 5$ & 5 \\
\hline Assign $_{6}$ & $\mathrm{Map}_{3}$ & Reduce $_{2}$ & $v 6, v 7, v 8, v 9, v 10$ & 5 \\
\hline $\operatorname{Assign}_{7}$ & Reduce $_{1}$ & $\mathrm{Map}_{4}$ & $v 1, v 2, v 3, v 4, v 5$ & 5 \\
\hline Assign $_{8}$ & Reduce $_{2}$ & $\mathrm{Map}_{5}$ & $v 6, v 7, v 8, v 9, v 10$ & 5 \\
\hline Assign $_{9}$ & $\mathrm{Map}_{4}$ & Reduce $_{3}$ & $\begin{array}{c}(v 1, v 2),(v 1, v 3),(v 3, v 4) \\
(v 3, v 5)\end{array}$ & 4 \\
\hline $\operatorname{Assign}_{10}$ & $\mathrm{Map}_{4}$ & Reduce $_{4}$ & $\begin{array}{c}(v 3, v 6),(v 4, v 6),(v 4, v 8) \\
(v 6, v 7)\end{array}$ & 4 \\
\hline $\operatorname{Assign}_{11}$ & $\mathrm{Map}_{5}$ & Reduce $_{4}$ & $\begin{array}{c}(v 3, v 6),(v 4, v 6),(v 4, v 8) \\
(v 6, v 7)\end{array}$ & 4 \\
\hline Assign $_{12}$ & $\mathrm{Map}_{5}$ & Reduce 5 & $\begin{array}{c}(v 7, v 8),(v 8, v 9),(v 8, v 10) \\
(v 7, v 10)\end{array}$ & 4 \\
\hline $\operatorname{Assign}_{13}$ & Reduce $_{3}$ & $\operatorname{Map}_{6}$ & $\begin{array}{c}(v 1, v 2),(v 1, v 3),(v 3, v 4) \\
(v 3, v 5)\end{array}$ & 4 \\
\hline $\operatorname{Assign}_{14}$ & Reduce $_{4}$ & $\mathrm{Map}_{7}$ & $\begin{aligned}(v 3, v 6), & (v 4, v 6),(v 4, v 8) \\
& (v 6, v 7)\end{aligned}$ & 4 \\
\hline $\operatorname{Assign}_{15}$ & Reduce $_{5}$ & $\mathrm{Map}_{8}$ & $\begin{array}{c}(v 7, v 8),(v 8, v 9),(v 8, v 10) \\
(v 7, v 10)\end{array}$ & 4 \\
\hline $\operatorname{Assign}_{16}$ & $\mathrm{Map}_{6}$ & Reduce $_{6}$ & $v 1, v 2, v 3, v 4, v 5$ & 5 \\
\hline $\operatorname{Assign}_{17}$ & $\mathrm{Map}_{7}$ & Reduce $_{6}$ & $v 1, v 2, v 3, v 4, v 5$ & 5 \\
\hline $\operatorname{Assign}_{18}$ & $\mathrm{Map}_{7}$ & Reduce $_{7}$ & $v 6, v 7, v 8, v 9, v 10$ & 5 \\
\hline $\operatorname{Assign}_{19}$ & $\mathrm{Map}_{8}$ & Reduce $_{7}$ & $v 6, v 7, v 8, v 9, v 10$ & 5 \\
\hline $\operatorname{Assign}_{20}$ & Reduce $_{6}$ & $\mathrm{Map}_{9}$ & $v 1, v 2, v 3, v 4, v 5$ & 5 \\
\hline $\operatorname{Assign}_{21}$ & Reduce $_{7}$ & $\operatorname{Map}_{10}$ & $v 6, v 7, v 8, v 9, v 10$ & 5 \\
\hline $\operatorname{Assign}_{22}$ & $\mathrm{Map}_{9}$ & Reduce $_{8}$ & $\begin{array}{c}(v 1, v 2),(v 1, v 3),(v 3, v 4) \\
(v 3, v 5)\end{array}$ & 4 \\
\hline $\operatorname{Assign}_{23}$ & $\mathrm{Map}_{9}$ & Reduce 9 & $\begin{array}{c}(v 3, v 6),(v 4, v 6),(v 4, v 8) \\
(v 6, v 7)\end{array}$ & 4 \\
\hline
\end{tabular}




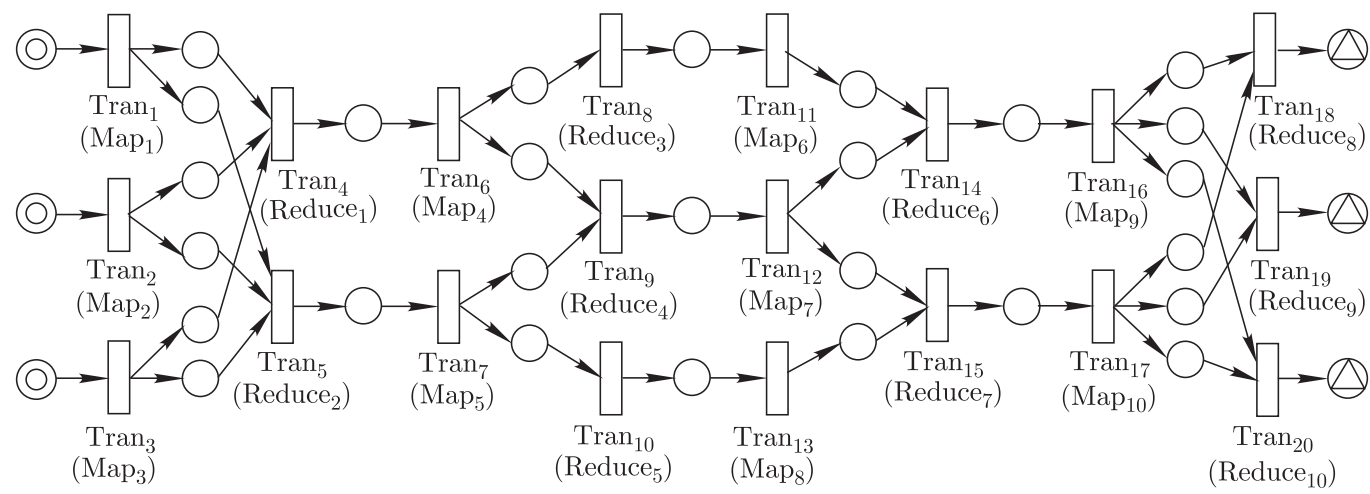

Fig. 4 OPN model of Case 2

Table 3 Assignments of Case 2

\begin{tabular}{|c|c|c|c|c|}
\hline$A S$ & From & To & Keys & |Keys| \\
\hline $\operatorname{Assign}_{24}$ & $\mathrm{Map}_{9}$ & Reduce $_{10}$ & $\begin{array}{c}(v 7, v 8),(v 8, v 9),(v 8, v 10) \\
(v 7, v 10)\end{array}$ & 4 \\
\hline $\operatorname{Assign}_{25}$ & $\operatorname{Map}_{10}$ & Reduce $_{8}$ & $\begin{array}{c}(v 1, v 2),(v 1, v 3),(v 3, v 4) \\
(v 3, v 5)\end{array}$ & 4 \\
\hline $\operatorname{Assign}_{26}$ & $\operatorname{Map}_{10}$ & Reduce $_{9}$ & $\begin{array}{c}(v 3, v 6),(v 4, v 6),(v 4, v 8) \\
(v 6, v 7)\end{array}$ & 4 \\
\hline $\operatorname{Assign}_{27}$ & $\operatorname{Map}_{10}$ & Reduce $10_{10}$ & $\begin{array}{c}(v 7, v 8),(v 8, v 9),(v 8, v 10) \\
(v 7, v 10)\end{array}$ & 4 \\
\hline
\end{tabular}

According to the approach presented in Section 5.1, we model the MapReduce procedures of Case 1 and Case 2 with OPN, as shown in Fig. 2 and Fig. 4, respectively.

\section{Identifying the rationality criteria}

We evaluate the rationality of MapReduce by analyzing the running situations of the resulting OPN model. To facilitate identifying the rationality criteria, we introduce the following definitions about OPN.

Definition 3 The triggering condition of a transition

For any transition $\operatorname{Tran}_{t}$, the number of tokens in each anterior place of $\operatorname{Tran}_{t}$ equals the token capacity of this place. Moreover, the number of tokens in each posterior place of $\operatorname{Tran}_{t}$ equals 0 .

$\operatorname{Tran}_{t}$ is triggered to execute only if it satisfies the triggering condition. The execution result of $\operatorname{Tran}_{t}$ is that the number of tokens in each anterior place of $\operatorname{Tran}_{t}$ equals 0 , and the number of tokens in each posterior place of $\operatorname{Tran}_{t}$ equals the token capacity of this place. In this way, tokens are migrated from the anterior places to the posterior places of $\operatorname{Tran}_{t}$ and trigger the execution of $\operatorname{Tran}_{t}$. The execution of $\operatorname{Tran}_{t}$ keeps an OPN model running and getting new states. The OPN model stops running as long as there is no triggered transition.

Definition 4 Reachable transitions

For any two transitions $\operatorname{Tran}_{t 1}$ and $\operatorname{Tran}_{t 2}$, if the execution of $\operatorname{Tran}_{t 1}$ makes the triggering condition of $\operatorname{Tran}_{t 2}$ hold, $\operatorname{Tran}_{t 2}$ is called a sequential transition of $\operatorname{Tran}_{t 1}$.
If there are multiple serial transitions between $\operatorname{Tran}_{t 1}$ and $\operatorname{Tran}_{t 2}, \operatorname{Tran}_{t 2}$ is regarded as a reachable transition from $\operatorname{Tran}_{t 1}$. Moreover, the anterior or posterior places of $\operatorname{Tran}_{t 2}$ are reachable from those of $\operatorname{Tran}_{t 1}$.

Definition 5 Conflicting transitions

Any two or more transitions with common anterior places are called conflicting transitions.

Definition 6 Weighted adjacency matrix

The matrix recording the weight value for each pair of sequential transitions is called the weighted adjacency matrix.

Definition 7 Weighted reachability matrix

The matrix recording the weight value for each pair of reachable transitions is called the weighted reachability matrix.

\subsection{Approach to identifying a straggler}

Since a straggler task has much longer execution time than other tasks, we identify a potential straggler task according to the execution time of its corresponding sub-object. The execution time of a sub-object only considers the operational transitions and their anterior and posterior places, which represent the operations of a Map or Reduce task in a sub-object.

Let Place $_{p}$. Capacity denote the maximum number of tokens that Place $p$ can hold. Let $\operatorname{Tran}_{y}$ denote the $y$ th operational transition in a sub-object, where $y \in Y$ derived by (1). Let $G_{y}$ and $H_{y}$ denote the numbers of anterior and posterior places of $\operatorname{Tran}_{y}$, respectively. Let $\operatorname{Tran}_{y}$.foreToken and $\operatorname{Tran}_{y}$.postToken denote the total token capacities of the anterior and posterior places of $\operatorname{Tran}_{y}$, respectively. We can derive

$$
\begin{aligned}
& \operatorname{Tran}_{y} \cdot \text { foreToken }=\sum_{g=1}^{G_{y}} \text { Place }_{g} \cdot \text { Capacity } \\
& \operatorname{Tran}_{y} \cdot \text { postToken }=\sum_{h=1}^{H_{y}} \text { Place }_{g} \cdot \text { Capacity. }
\end{aligned}
$$


Assume the arrival process of tokens at the anterior or posterior places of a transition in an OPN model follows the Poisson distribution. Let $\lambda_{1}$ and $\lambda_{2}$ denote the arrival rates of tokens at the anterior and posterior places of an operational transition, respectively. Moreover, $\lambda_{1}$ equals the arrival rate of tokens at the posterior places of every transition in the high level OPN. The values of $\lambda_{1}$ and $\lambda_{2}$ depend on different applications. We use $\lambda_{1}$ and $\lambda_{2}$ to simulate the amount of input data. Larger amounts of input data need more processing and read-write time. Hence, the values of $\lambda_{1}$ and $\lambda_{2}$ decrease with the growth of input data.

Theorem 1 Let $\operatorname{Tran}_{y}$. Time denote the execution time of the $y$ th operational transition in a sub-object, then $\operatorname{Tran}_{y}$. Time is given by

$$
\operatorname{Tran}_{y} \cdot \text { Time }=\frac{\operatorname{Tran}_{y} \cdot \text { postToken }}{\lambda_{2}} .
$$

Proof During the arrival process of tokens at the posterior places of $\operatorname{Tran}_{y}$, the interval time between two tokens is $\frac{1}{\lambda_{2}}$. The execution of $\operatorname{Tran}_{y}$ cannot be accomplished until all the $\operatorname{Tran}_{y}$.postToken tokens have moved to the posterior places of $\operatorname{Tran}_{y}$. Hence, the execution time of $\operatorname{Tran}_{y}$ is $\frac{\operatorname{Tran}_{y} \cdot \operatorname{postToken}}{\lambda_{2}}$. Thus Theorem 1 is proved.

Theorem 2 Let SubObject ${ }_{s}$. Time denote the execu-

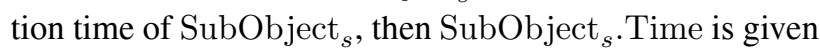
by

$$
\begin{gathered}
\text { SubObject }_{s} \cdot \text { Time }= \\
\max _{0<y \leqslant Y}\left\{\frac{\operatorname{Tran}_{y} \cdot \text { foreToken }}{\lambda_{2}}+\frac{\operatorname{Tran}_{y} \cdot \text { postToken }}{\lambda_{2}}\right\} .
\end{gathered}
$$

Proof According to Definition 3, $\operatorname{Tran}_{y}$ cannot be performed until all the $\operatorname{Tran}_{y}$.foreToken tokens have arrived to its anterior places. The time taken by this arrival process is $\frac{\operatorname{Tran}_{y} \text {.foreToken }}{\lambda_{1}}$. Thus, according to Theorem 1, the total transmission time of tokens from the anterior places of $\operatorname{Tran}_{y}$ to its posterior places is $\frac{\operatorname{Tran}_{y} \text {.foreToken }}{\lambda_{1}}+$ $\frac{\operatorname{Tran}_{y} \text {. postToken }}{\lambda_{2}}$. Since all the operational transitions are parallel, the execution time of SubObject ${ }_{s}$ equals the longest transmission time of those operational transitions. Thus Theorem 2 is proved.

For MapReduce, a straggler is a relative concept. During a Map or Reduce phase, a straggler takes relatively much longer time than other normal tasks. However, the execution time of a straggler in a MapReduce procedure may be equal to or even shorter than that of a normal task in another MapReduce procedure. Consequently, in order to identify a potential straggler, its execution time should be compared with a threshold time, which can be set by program designers according to concrete applications.

Let $\tau$ denote the threshold time for identifying the stragglers. If the execution time of SubObject ${ }_{s}$ is larger than $\tau$, the original Map or Reduce task denoted by SubObject ${ }_{s}$ may be a potential straggler.

The aforementioned three parameters, namely $\lambda_{1}, \lambda_{2}$ and the threshold $\tau$, are assumed to be set by program designers. Their values are obtained by experience or from historical statistics, and vary with different applications. Except these three parameters, all the other parameters are directly derived from the formulas proposed by the paper, namely from the predefined MapReduce procedures indirectly.

\subsection{Approach to identifying a Map conflict}

In Section 5.1, conflicting Map assignments are transformed into the same place, which is the common anterior place of two or more transitions. Since conflicting transitions are caused by common anterior places, we disclose Map conflicts through the conflicting transitions that emerge during the execution of OPN.

Theorem 3 Conflicting transitions stop the execution of OPN.

Proof Suppose two transitions $\operatorname{Tran}_{t 1}$ and $\operatorname{Tran}_{t 2}$ are conflicting transitions, and $\mathrm{Place}_{p}$ is their common anterior place. If $\operatorname{Tran}_{t 1}$ has been executed, according to Defi-

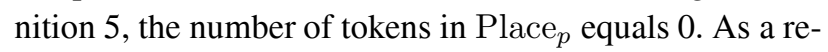
sult, $\operatorname{Tran}_{t 2}$ cannot be performed in this case. For the same reason, $\operatorname{Tran}_{t 1}$ cannot be performed when $\operatorname{Tran}_{t 2}$ has been executed. Thus Theorem 3 is proved.

We can easily find out the conflicting transitions during the execution of the resulting OPN model according to Theorem 3. These transitions are transformed from the Reduce tasks, which receive conflicting Map assignments in the original MapReduce procedure.

\subsection{Approach to identifying the execution time}

The execution time of MapReduce depends on the program design, the amount of data to be processed, the network performance, and some other factors. However, the execution time of OPN reflects the rationality of MapReduce design in terms of time spending. We employ the execution time of OPN to simulate the possible execution time of MapReduce from a MapReduce design point of view.

Similar to the approach to identifying stragglers, we use $\lambda_{1}$, the arrival rate of tokens at the posterior places of every transition in the high level OPN, to simulate the amount of input data.

Let $\boldsymbol{U}=\left(u_{i, j}\right)$ denote the weighted adjacency matrix of 
the transitions and sub-objects in an OPN model. If the intersection of $\mathrm{TrOb}_{i}$. postPlace and $\mathrm{TrOb}_{j}$. forePlace is an empty set, the value of $u_{i, j}$ equals 0 . Otherwise the value of $u_{i, j}$ is given by

$$
u_{i j}=\operatorname{TrOb}_{i} . \text { Time }+\operatorname{TrOb}_{j} \text {.Time }
$$

where $0<i \leqslant M+R$ and $0<j \leqslant M+R$. This matrix records the total execution time of any two sequential TrObs. The execution time of a sub-object can be given by (5). Similar to Theorem 1, the execution time of a transition, denoted by $\operatorname{Tran}_{t}$, in the high level OPN can be given by

$$
\operatorname{Tran}_{t} . \text { Time }=\frac{\operatorname{Tran}_{t} \cdot \text { postToken }}{\lambda_{1}} .
$$

Let $\boldsymbol{V}=\left(v_{i, j}\right)$ denote the weighted reachability matrix of the transitions and sub-objects in an OPN model. It records the total execution time of transitions and subobjects, which are sequentially executed from $\mathrm{TrOb}_{i}$ to $\mathrm{TrOb}_{j}$. Based on the Floyd algorithm [25], we define $v_{i, j}$ in Algorithm 2, where $u_{i, k}+u_{k, j}-\mathrm{TrOb}_{k}$. Time denotes the sum of $\mathrm{TrOb}_{i}$. Time, $\mathrm{TrOb}_{j}$. Time and $\mathrm{TrOb}_{k}$. Time. To find the value of $v_{i, j}$, Algorithm 2 traverses each transition and each sub-object from $\mathrm{TrOb}_{i}$ to $\mathrm{TrOb}_{j}$ and accumulates the corresponding value in the weighted adjacency matrix. Its time complexity is $O\left((M+R)^{3}\right)$, implying that this algorithm can be accomplished in polynomial time.

Algorithm 2 Create matrix $\boldsymbol{V}$

01: for $k=1 ; k \leqslant M+R ; k++$

02: for $i=1 ; i \leqslant M+R ; i++$

03: $\quad$ for $j=1 ; j \leqslant M+R ; j++$

04: $\quad v_{i, j}=u_{i, j}=\max \left\{u_{i, j}, u_{i, k}+u_{k, j}-\right.$ $\mathrm{TrOb}_{k}$.Time\};

Theorem 4 Let OPN.Time denote the execution time of OPN, then OPN.Time is given by

$$
\text { OPN.Time }=\max _{\substack{0<i \leqslant M+R \\ 0<j \leqslant M+R}}\left\{v_{i, j}\right\} .
$$

Proof Suppose the maximum value of the elements of matrix $\boldsymbol{V}$ is not the execution time of OPN. Then there must be a list of reachable transitions and sub-objects, whose total execution time is larger than $\max \left\{v_{i, j}\right\}$. This is contrary to the definition of matrix $\boldsymbol{V}$. Thus Theorem 4 is proved.

We compare OPN.Time with the value that the designer or user can accept, so as to identify the rationality of MapReduce design from the aspect of time spending.

\section{Evaluation}

\subsection{Modelling and simulation environment of OPN}

Our research group has built a modelling and simulation environment of OPN, named OPMSE. It is used for simulating the operations and procedures of large-scale complex systems by executing OPN models.

OPMSE consists of two main modules. The first one is the modelling module. It reads the XML or binary file of an OPN model to generate a corresponding graph form. It supports users to directly build graph forms for OPN models. The second one is the simulation module. It executes OPN models and analyzes corresponding results. It allows users to set a particular time distribution for the transfer of tokens between transitions.

We transform the MapReduce procedures of the two cases into corresponding OPN models, saved as XML files. Then we generate their graphic formats as shown in Fig. 2 and Fig. 4, and set the token arrival rate for each place to simulate the generation time of the keys of intermediate data, and run the procedures with OPNMSE, so as to analyze their rationality through the running process.

\subsection{Rationality verification}

According to the rationality criteria proposed in Section 3, we analyze the running situations of the OPN models for Case 1 and Case 2 as follows.

\subsubsection{Identifying potential stragglers}

We use Case 1 to illustrate how to identify potential stragglers. The types of keys processed by Reduce 1 are more than that of the other Reduce tasks. We transform Reduce 1 into a sub-object, denoted by SubObject ${ }_{1}$, to determine whether Reduce ${ }_{1}$ is a potential straggler. Let the threshold number $C$ be 5. Let $\mid$ Reduce $_{1}$.Keys $\mid$ denote the number of types of intermediate keys processed by Reduce ${ }_{1}$. We derive the ceiling of $C$ dividing $\mid$ Reduce $_{1}$.Keys $\mid$ is 3. According to Section 5.2, there should be three operational


of the OPN model transformed from the MapReduce procedure of Case 1. Fig. 5(b) shows the structure of the lower level OPN model denoted by SubObject . $_{\text {. }}$

Table 2 indicates that there are twelve types of keys in every input assignment of Reduce ${ }_{1}$, which has no output assignment. Therefore, the token capacity of each input place in SubObject $_{1}$ is 12 , and the token capacity of the only output place in SubObject ${ }_{1}$ is 1 . According to Section 5.2, the token capacities of an anterior place and a posterior place of an operational transition are 4 and 1 , respectively. Each operational transition has three anterior places and one posterior place. Hence, for SubObject, there is $\operatorname{Tran}_{1}$.foreToken $=\operatorname{Tran}_{2}$.foreToken $=$ $\operatorname{Tran}_{3}$.foreToken $=12$ and $\operatorname{Tran}_{1}$.postToken $=$ $\operatorname{Tran}_{2} \cdot$ postToken $=\operatorname{Tran}_{3} \cdot$ postToken $=1$, according to (2) and (3). 


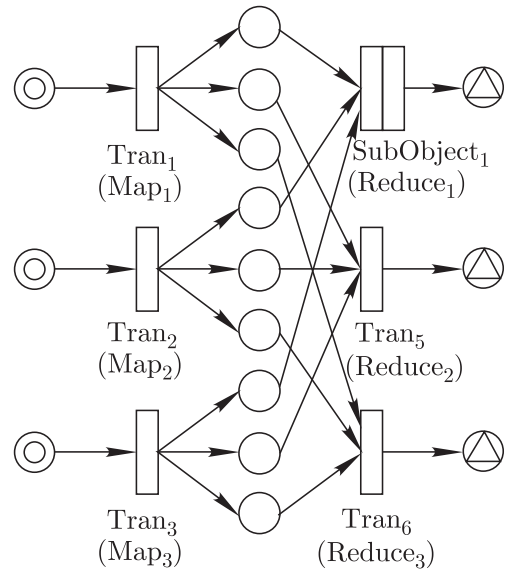

(a) High level OPN model of Case 1

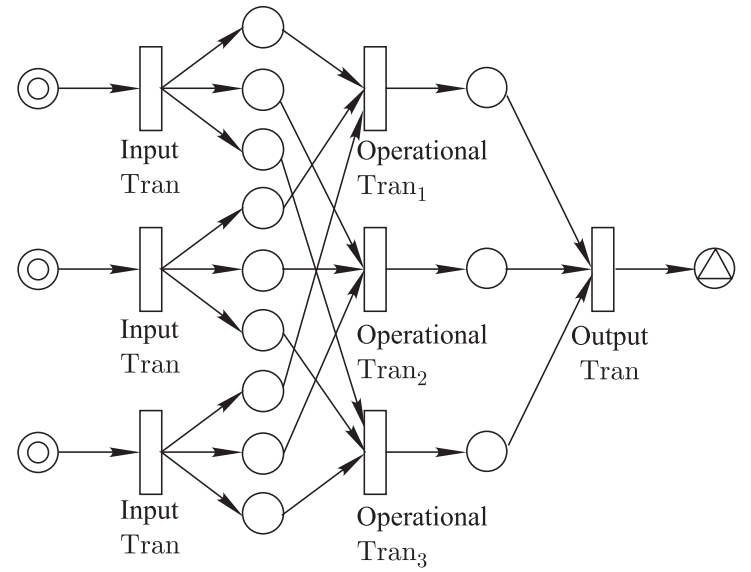

(b) Low level OPN model denoted by SubObject1

Fig. 5 OPN model of Case 1 for identifying the stragglers

Fig. 6 shows the variation of the execution time of SubObject ${ }_{1}$ with the increase of $\lambda_{2}$ by fixing $\lambda_{1}$. We only need to define the values of $\lambda_{1}$ and $\lambda_{2}$, OPMSE will calculate the execution time of SubObject ${ }_{1}$. We set a threshold value $\tau$, represented by the dotted line in Fig. 6, to identify that Reduce $_{1}$ is a potential straggler when the execution time of SubObject ${ }_{1}$ is less than $\tau$.

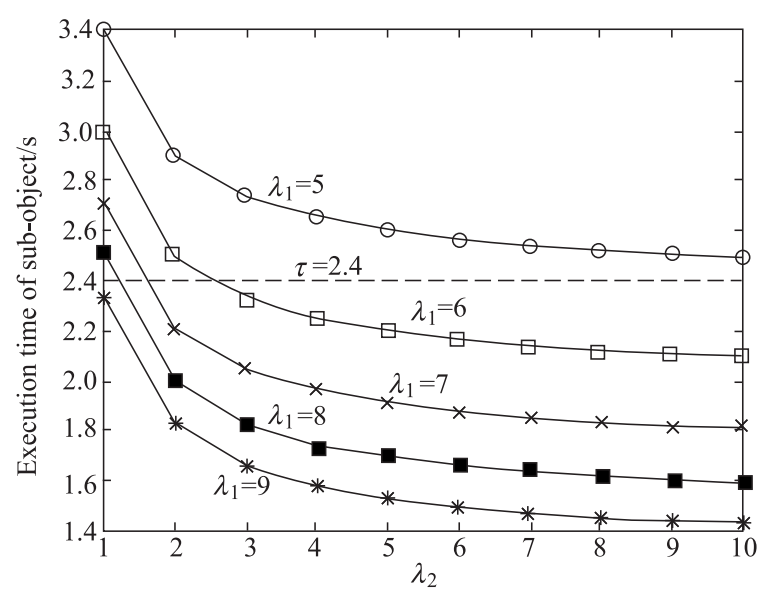

Fig. 6 Execution time of SubObject S $_{1}$

\subsubsection{Identifying Map conflicts}

We use Case 2 to illustrate how to identify Map conflicts, which can be classified into two categories. The first one is that the conflicting assignments are sent by the same Map task. Assume that the key $\left(v_{3}, v_{4}\right)$ of Assign $_{9}$ is designed as $\left(v_{3}, v_{6}\right)$ which is a key of Assign $_{10}$ by mistake. Thus, Assign $_{9}$ and Assign 10 , both sent by Map 4 , become a Map conflict. The second one is that the conflicting assignments are sent by different Map tasks. Assume that the key $v_{5}$ of Assign $_{16}$ is designed as $v_{6}$ which is a key of Assign 18 by mistake. Then Assign $_{16}$ and Assign 18 , separately sent by $\mathrm{Map}_{6}$ and $\mathrm{Map}_{7}$, become a Map conflict. Fig. 7 shows the graph form of the OPN model transformed from the MapReduce procedure of Case 2 with the two kinds of Map conflicts.

We use OPMSE to execute the OPN model of Case 2 with above Map conflicts. The execution stops at $\operatorname{Tran}_{6}$, which is transformed from $\mathrm{Map}_{4}$. When we correct the key $\left(v_{3}, v_{6}\right)$ to $\left(v_{3}, v_{4}\right)$ for Assign $_{9}$, the execution stops at $\operatorname{Tran}_{11}$ and $\operatorname{Tran}_{12}$, which are transformed from $\mathrm{Map}_{6}$ and $\mathrm{Map}_{7}$, respectively.

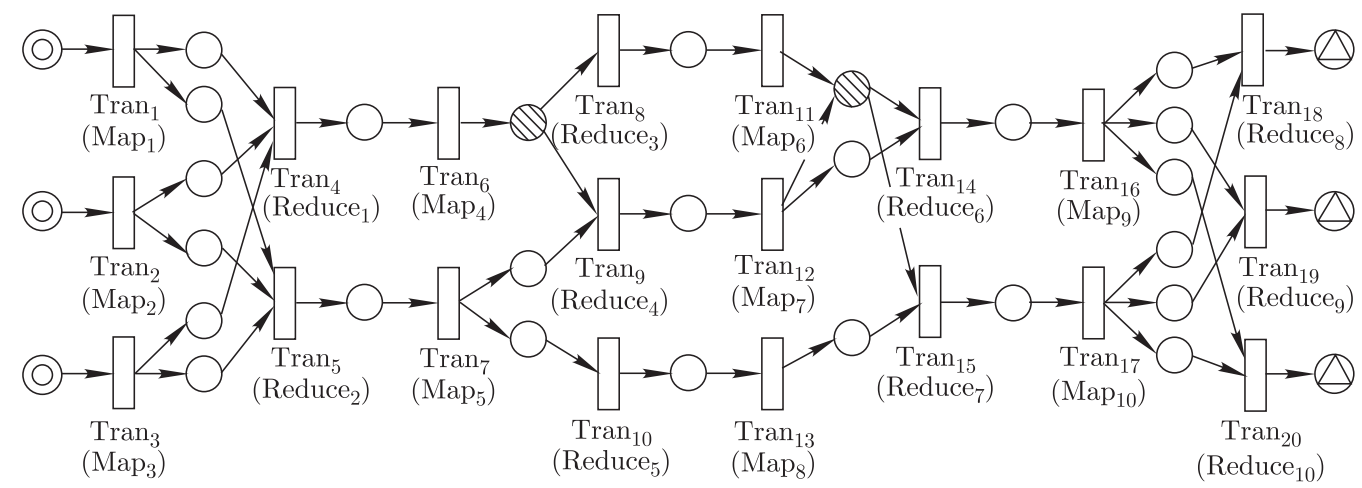

Fig. 7 OPN of Case 2 for judging the Map conflicts 
When we change the key $v_{6}$ to $v_{5}$ for $\operatorname{Assign}_{16}$, the OPN model of Case 2 is executed successfully.

\subsubsection{Identifying execution time}

If a MapReduce procedure is logically executable, we can simulate its possible execution time by executing its OPN model. We utilize the OPN models in Fig. 2 and Fig. 4 to illustrate how to simulate the execution time. In this simulation, we assume $\lambda_{1}=1$.

In Case 1, according to (7), we derive that $\operatorname{Tran}_{1} \cdot$ Time $=\operatorname{Tran}_{2}$. Time $=\operatorname{Tran}_{3}$. Time $=22 \mathrm{~s}$ and $\operatorname{Tran}_{4}$. Time $=\operatorname{Tran}_{5}$. Time $=\operatorname{Tran}_{6}$. Time $=1 \mathrm{~s}$. Let $\boldsymbol{U}_{1}$ denote the weighted adjacent matrix of the transitions of Case 1. According to (6), we derive

$$
\boldsymbol{U}_{1}=\left[\begin{array}{cccccc}
0 & 0 & 0 & 23 & 23 & 23 \\
0 & 0 & 0 & 23 & 23 & 23 \\
0 & 0 & 0 & 23 & 23 & 23 \\
0 & 0 & 0 & 0 & 0 & 0 \\
0 & 0 & 0 & 0 & 0 & 0 \\
0 & 0 & 0 & 0 & 0 & 0
\end{array}\right]
$$

Let $V_{1}$ denote the weighted reachability matrix of the transitions of Case 1. According to Algorithms 1 and 2, we derive that $\boldsymbol{V}_{1}$ equals $\boldsymbol{U}_{1}$. Thus, according to (8), the execution time of Case 1 is $23 \mathrm{~s}$. When $\lambda_{1}$ increases from 1 to 10 , the execution time of Case 1 decreases to $2.3 \mathrm{~s}$.

In Case 2, according to (7), we derive that $\operatorname{Tran}_{1}$. Time $=\operatorname{Tran}_{2}$. Time $=\operatorname{Tran}_{3}$.Time $=$ $\operatorname{Tran}_{12}$. Time $=10 \mathrm{~s}$, $\operatorname{Tran}_{4}$. Time $=\operatorname{Tran}_{5}$. Time $=$ $\operatorname{Tran}_{11}$.Time $=\operatorname{Tran}_{13}$.Time $=\operatorname{Tran}_{14}$. Time $=$ $\operatorname{Tran}_{15} \cdot$ Time $=5 \mathrm{~s}, \operatorname{Tran}_{6} \cdot$ Time $=\operatorname{Tran}_{7} \cdot$ Time $=8 \mathrm{~s}$, Tran $_{8}$. Time $=$ Tran $_{9}$.Time $=\operatorname{Tran}_{10}$. Time $=$ $4 \mathrm{~s}, \operatorname{Tran}_{16}$. Time $=\operatorname{Tran}_{17}$. Time $=12 \mathrm{~s}$, and $\operatorname{Tran}_{18} \cdot$ Time $=\operatorname{Tran}_{19}$. Time $=\operatorname{Tran}_{20}$. Time $=1 \mathrm{~s}$. Let $\boldsymbol{U}_{2}$ denote the weighted adjacent matrix of the transitions of Case 2. According to (6), we derive

$$
\boldsymbol{U}_{2}=\left[\begin{array}{cccccccccc}
0 & 0 & 0 & 15 & 15 & 0 & \cdots & 0 & 0 & 0 \\
0 & 0 & 0 & 15 & 15 & 0 & \cdots & 0 & 0 & 0 \\
0 & 0 & 0 & 15 & 15 & 0 & \cdots & 0 & 0 & 0 \\
0 & 0 & 0 & 0 & 0 & 13 & \cdots & 0 & 0 & 0 \\
\vdots & \vdots & \vdots & \vdots & \vdots & \vdots & \ddots & \vdots & \vdots & \vdots \\
0 & 0 & 0 & 0 & 0 & 0 & \cdots & 13 & 13 & 13 \\
0 & 0 & 0 & 0 & 0 & 0 & \cdots & 13 & 13 & 13 \\
0 & 0 & 0 & 0 & 0 & 0 & \cdots & 0 & 0 & 0 \\
0 & 0 & 0 & 0 & 0 & 0 & \cdots & 0 & 0 & 0 \\
0 & 0 & 0 & 0 & 0 & 0 & \cdots & 0 & 0 & 0
\end{array}\right] .
$$

Let $V_{2}$ denote the weighted reachability matrix of the transitions of Case 2. According to Algorithms 1 and 2, we derive

$$
\boldsymbol{V}_{2}=\left[\begin{array}{cccccccccc}
0 & 0 & 0 & 15 & 15 & 23 & \cdots & 55 & 55 & 55 \\
0 & 0 & 0 & 15 & 15 & 23 & \cdots & 55 & 55 & 55 \\
0 & 0 & 0 & 15 & 15 & 23 & \cdots & 55 & 55 & 55 \\
0 & 0 & 0 & 0 & 0 & 13 & \cdots & 45 & 45 & 45 \\
\vdots & \vdots & \vdots & \vdots & \vdots & \vdots & \ddots & \vdots & \vdots & \vdots \\
0 & 0 & 0 & 0 & 0 & 0 & \cdots & 13 & 13 & 13 \\
0 & 0 & 0 & 0 & 0 & 0 & \cdots & 13 & 13 & 13 \\
0 & 0 & 0 & 0 & 0 & 0 & \cdots & 0 & 0 & 0 \\
0 & 0 & 0 & 0 & 0 & 0 & \cdots & 0 & 0 & 0 \\
0 & 0 & 0 & 0 & 0 & 0 & \cdots & 0 & 0 & 0
\end{array}\right]
$$

Here, we omit some of the details of $\boldsymbol{U}_{2}$ and $\boldsymbol{V}_{2}$ for the space limitation. According to (8), the execution time of Case 2 is $55 \mathrm{~s}$. When $\lambda_{1}$ increases from 1 to 10 , the execution time of Case 2 decreases to $5.5 \mathrm{~s}$.

Fig. 8 shows the variation of the execution time of Case 1 and Case 2 with the increase of $\lambda_{1}$.

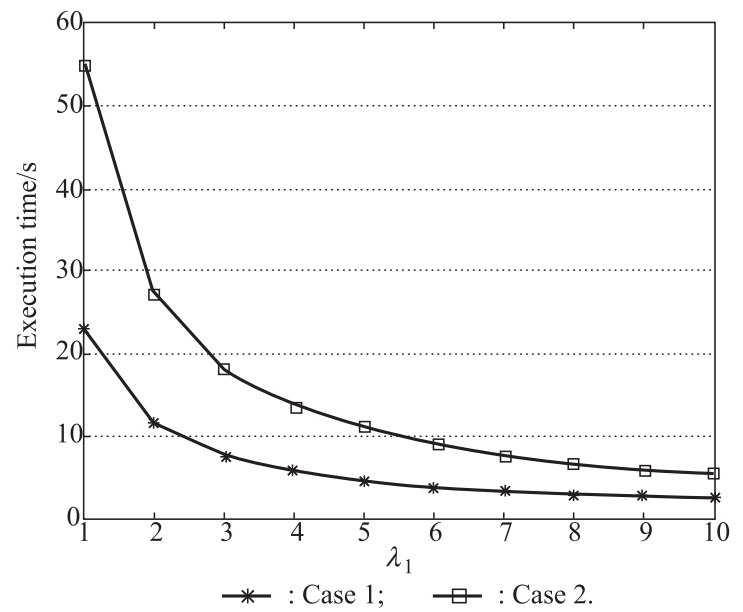

Fig. 8 Execution time of Case 1 and Case 2

\section{Conclusions}

The rationality verification process indicates that our method can effectively verify the irrational problems of MapReduce.

Without regard to hardware problems, we derive that a straggler in an application might be a normal task in another application, because different applications have different amounts of input data. To identify the second rationality criterion, we use the arrival rate of tokens to simulate the amount of input data. Fig. 6 shows that the execution time of the sub-object decreases with the increase of $\lambda_{1}$ and $\lambda_{2}$. By changing the values of $\lambda_{1}$ and $\lambda_{2}$, our approach can flexibly identify the potential stragglers in a MapReduce procedure for different applications.

According to the execution rule of OPN, one normal place can connect with only two transitions. Fig. 7 indicates that the conflicting Map tasks are transformed into a 
normal place connecting with at least three transitions. It makes the execution of OPN stop at the transition whose posterior includes this normal place. In such a way, we can easily identify the hidden Map conflicts in a complex MapReduce procedure.

We use the execution time of OPN to simulate that of MapReduce from the perspective of its design. Similar to the determination of stragglers, the execution time of the same MapReduce procedure can vary for different input data. Fig. 8 shows that the execution time of the OPN models of the two cases decreases with the increase of $\lambda_{1}$. By changing the values of $\lambda_{1}$, our approach can effectively identify the third rationality criterion for different applications.

\section{References}

[1] DEAN J, GHEMAWAT S. MapReduce: simplified data processing on large clusters. Proc. of the 6th USENIX Conference on Operating System Design and Implementation, 2004: $137-150$.

[2] CHANG F, DEAN J, GHEMAWAT S, et al. Bigtable: a distributed storage system for structured data. Proc. of the 7th USENIX Conference on Operating Systems Design and Implementation, 2006: 205-218.

[3] ISARD M, BUDIU M, YU Y, et al. Dryad: distributed dataparallel programs from sequential building blocks. Proc. of the 21st ACM Symposium on Operating Systems Principles, 2007: 59-72.

[4] Open source software for creating private and public clouds. http://www.openstack.org. Apache hadoop. http://hadoop. apache.org.

[5] LIN J. The curse of Zipf and limits to parallelization: a look at the stragglers problem in MapReduce. Proc. of the 7th ACM Workshop on Large-Scale Distributed Systems for Information Retrieval, 2009: 57-62.

[6] WU D, XIA Y, SUN X S, et al. Masking failures from application performance in data center networks with shareable backup. Proc. of the International Conference on Communication Architectures, Protocols \& Apps, 2018: 176-190.

[7] CHEN L, LINGYS J, CHEN K, et al. AuTO: scaling deep reinforcement learning for datacenter-scale automatic traffic optimization. Proc. of the International Conference on Communication Architectures, Protocols \& Apps, 2018: 191-205.

[8] MOTAMENI H, MOVAGHAR A, SHIRAZI B, et al. Analysis software with an object-oriented Petri net model. World Applied Sciences Journal, 2008, 3(4): $565-576$.

[9] HONG J, BAE D. Software modeling and analysis using a hierarchical object-oriented Petri net. Information Sciences, 2000, 130(1/4): $133-164$.

[10] SANDHOLM T, LAI K. MapReduce optimization using regulated dynamic prioritization. Proc. of the International Conference on Measurement and Modeling of Computer Systems, 2009: 299-310.

[11] RANGER C, RAGHURAMAN R, PENMETSA A, et al. Evaluating MapReduce for multi-core and multiprocessor systems. Proc. of the 13th IEEE International Symposium on High-Performance Computer Architecture, 2007: 13 - 24.

[12] LAMMEL R. Google's MapReduce programming model re- visited. Elsevier Science of Computer Programming, 2008, 70(1): $1-30$.

[13] ZAHARIA M, KONWINSKI A, JOSEPH A D, et al. Improving MapReduce performance in heterogeneous environments. Proc. of the 8th USENIX Conference on Operating Systems Design and Implementation, 2008: 29-42.

[14] KAVULYA S, TAN J, GANDHI R, et al. An analysis of traces from a production MapReduce cluster. Pittsburgh, USA: Parallel Data Laboratory at Carnegie Mellon University, 2009.

[15] REN X, ANANTHANARAYANAN G, WIERMAN A, et al. Hopper: decentralized speculation-aware cluster scheduling at scale. Proc. of the International Conference on Communication Architectures, Protocols \& Apps, 2015: 379-392.

[16] WEI W, DU J, YU T, et al. SecureMR: a service integrity assurance framework for MapReduce. Proc. of the IEEE Annual Computer Security Applications Conference, 2009: 73-82.

[17] ZHU Y, KANG N, CAO J. Packet-level telemetry in large datacenter networks. Proc. of the International Conference on Communication Architectures, Protocols \& Apps, 2015: 479491.

[18] YANG H, DASDAN A, HSIAO R L, et al. Map-ReduceMerge: simplified relational data processing on large clusters. Proc. of the International Conference on Management of Data, 2007: $1029-1040$.

[19] KRUIJF M, SANKARALINGAM K. MapReduce for the Cell B.E. architecture. IBM Journal of Research and Development, 2009, 53(5): $747-758$.

[20] DEAN J, GHEMAWAT S. MapReduce: a flexible data processing tool. Communications of the ACM, 2010, 53(1): $72-$ 77.

[21] HUANG B, ZHOU M, ZHANG P, et al. Speedup techniques for multi-objective integer programs in designing optimal and structurally simple supervisors of AMS. IEEE Trans. on Systems, Man and Cybernetics: Systems, 2018, 48(1): 77-88.

[22] HUANG B, ZHOU M C, HUANG Y S, et al. Supervisor synthesis for FMS based on critical activity places. IEEE Trans. on Systems, Man and Cybernetics: Systems, 2017, 49(5): 1-10.

[23] COHEN J. Graph twiddling in a MapReduce world. IEEE Computing in Science and Engineering, 2009, 2(4): 29-41.

[24] WEISSTEIN E. Floyd-Warshall algorithm. http://mathworld. wolfram.com/Floyd-WarshallAlgorithm.html.

\section{Biographies}



DING Zeliu was born in 1983. He is a Ph.D. and an assistant research fellow in Academy of Military Sciences. His research interests are data center networks, cloud computing and complex information systems.

E-mail: dingzeliu@gmail.com

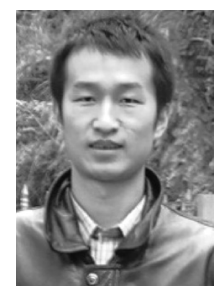

GUO Deke was born in 1980. He is a Ph.D. and a professor in National University of Defense Technology. His research interests are P2P, data center networks, cloud computing and mobile computing. E-mail: guodeke@gmail.com 


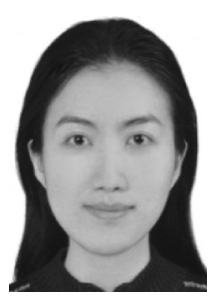

CHEN Xi was born in 1986. She is a Ph.D. in McGill University. Her research interests are cyberphysical systems and cloud computing.

E-mail: Chenxiwarm@gmail.com

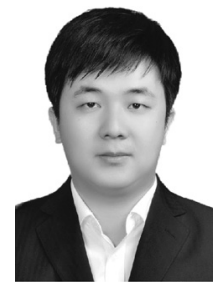

CHEN Jin was born in 1986. He is a lecturer in Naval University of Engineering. His research interests are underwater communication and picture processing.

E-mail: chenjin_198607@163.com 